\title{
SINOBAS y su relación con la predicción y vigilancia meteorológicas
}

https://doi.org/10.31978/639-19-010-0.079

\author{
Jesús Riesco Martín'1 (jriescom@aemet.es) \\ Delia Gutiérrez Rubio² (dgutierrezr@aemet.es) \\ Salvador Ponce Gutiérrez ${ }^{1}$ (sponceg@magrama.es)
}

${ }^{1}$ AEMET / Delegación Territorial en Andalucía / Centro Meteorológico de Málaga

${ }^{2}$ AEMET / Delegación Territorial en Andalucía

\begin{abstract}
RESUMEN
Cumplidos ya más de cinco años de uso operativo, el sistema SINOBAS de AEMET (Sistema de Notificación de Observaciones Atmosféricas Singulares) destaca como un componente fundamental de la estrategia de AEMET de crowdsourcing e internet de las cosas. Está operativo desde abril de 2013 y se está convirtiendo en una valiosa base de datos de fenómenos meteorológicos que, de otro modo, serían difícilmente localizables. Todos los reportes introducidos en el sistema son después validados por técnicos de AEMET y se les asigna una fiabilidad. SINOBAS también gestiona una cuenta de Twitter, @AEMET_SINOBAS, que es un canal ágil de comunicación con el público así como una vía de popularización del sistema y de seguimiento de la actualidad meteorológica.

La base de datos de SINOBAS permite disponer de un archivo de fenómenos singulares, y a veces adversos, que pueden ser utilizados para caracterizar los tipos de episodios conducentes a tales fenómenos, incidiendo en la mejora de la predicción. Asimismo la propia aplicación dispone de sendos buscadores en Twitter y en foros de meteorología, lo que le confiere un valor añadido como herramienta de primer escalón de vigilancia meteorológica.
\end{abstract}

PALABRAS CLAVE: base de datos; fenómenos singulares; vigilancia meteorológica; rastreo en Twitter; rastreo en foros.

\section{INTRODUCCIÓN}

Ciertos fenómenos atmosféricos que pueden considerarse raros o extraordinarios, a menudo violentos y súbitos y, en muchos casos, asociados a la convección, no tenían en el pasado un seguimiento rutinario en AEMET. La necesidad de disponer de un Sistema que recoja este tipo de fenómenos se basa en varias razones, entre otras:

- La necesidad imperiosa de dar respuesta a las demandas de la sociedad de acuerdo con el vigente ordenamiento jurídico. AEMET, como Autoridad Meteorológica del Estado, tiene encomendada la verificación de la Tempestad Ciclónica Atípica (BOE de 24 de febrero de 2004, Real Decreto 300/2004 y BOE de 27 de octubre de 2011, Real Decreto 1386/2011), lo que incluye fenómenos como tornados, recogidos en SINOBAS. El archivo de SINOBAS es por tanto una fuente de información que facilita en muchos casos el cumplimiento de este compromiso.

- La necesidad de verificar las predicciones que se realizan en AEMET. SINOBAS es una fuente de información muy útil para realizar verificaciones de los fenómenos recogidos en el Sistema, algunos de los cuales quedan dentro del marco del plan Meteoalerta de AEMET. 
- La necesidad de disponer de una base de datos de estos eventos para realizar estudios específicos, futuras climatologías, consideraciones sobre la influencia del cambio climático en el impacto de este tipo de fenómenos, etc.

- La conveniencia de aunar esfuerzos y colaborar con proyectos europeos similares, como la ESWD, European Severe Weather Database, http://www.essl.org/. La información recogida en SINOBAS es fácilmente compartible y, recíprocamente, nuestro Sistema puede asimilar datos de otras bases de datos.

En el Sistema SINOBAS se recoge y almacena información sobre fenómenos locales, poco frecuentes, de intensidad significativa y, en ocasiones, de alto impacto social, que no son fácilmente detectables por los medios convencionales de observación in situ o a distancia de AEMET, ni por redes similares de otros organismos, bien sea por la escasa densidad de la propia red de observación, bien por las limitaciones de los sistemas de teledetección actuales (satélite, radar, etc.), que no poseen las resoluciones espaciotemporales adecuadas. La principal fuente de información de SINOBAS es la observación reportada por cualquier ciudadano, ya sea directa o referenciada, convenientemente documentada, que es verificada posteriormente por AEMET.

SINOBAS corresponde al acrónimo de SIstema de Notificación de OBservaciones Atmosféricas Singulares, y es también un homenaje a Manuel Rico y Sinobas (1821-1898) por sus estudios pioneros de meteorología en España en el siglo XIX.

Además, hay que tener en cuenta las siguientes consideraciones legales, contenidas en la propia página de SINOBAS:

- La Agencia Estatal de Meteorología, AEMET, declina toda responsabilidad sobre los daños y perjuicios que pudieran ser ocasionados por la interpretación y el uso de la información puesta a disposición de los ciudadanos en esta página web.

- Su diseño, sus códigos fuente y sus contenidos pertenecen a AEMET y están protegidos por los correspondientes derechos de propiedad intelectual.

- La información contenida en esta página web se ofrece gratuitamente a los ciudadanos para que pueda ser utilizada libremente por ellos. No obstante, deberán mencionar a AEMET, cada vez que la utilicen para usos distintos del particular o privado.

- AEMET se reserva el derecho de añadir, modificar o eliminar la información contenida en esta página web. La introducción de datos e imágenes relativos a un evento implica que la información también pasa a ser propiedad de AEMET.

\section{FENÓMENOS METEOROLÓGICOS INCLUIDOS EN SINOBAS}

Los fenómenos recogidos en SINOBAS están asociados en su mayoría a una escala del orden de, o menor que, la convectiva (resolución espacial de unos pocos kilómetros y temporal del orden de minutos o unas pocas horas). No se tienen en cuenta fenómenos como un frente activo, una perturbación ciclónica de latitudes medias o de origen tropical, o incluso un derecho, línea de turbonada o sistema convectivo de mesoescala. Se consideran por separado los elementos individuales embebidos en dichos sistemas y no el sistema como un todo. De la misma manera, no se incluyen los vientos intensos o huracanados de origen orográfico conducidos sinóptica o mesoescalarmente como el cierzo, el mistral, etc., ni tampoco las galernas, la ciclogénesis mediterránea, ni fenómenos ligados a subidas o bajadas del mar generadas por tsunamis, aunque sí otros fenómenos marítimos raros más locales, como rissagas y elevaciones locales del nivel del mar. Sin embargo, sí se han incluido algunos fenómenos de escala menos reducida, como nevadas que cumplan ciertos requisitos de excepcionalidad y lluvia engelante. He aquí el listado detallado de fenómenos incluidos en el Sistema: 
- Tornados, Trombas marinas y Tubas. Los tornados, trombas y tubas se consideran «singulares» en todos los casos, sin que se haya establecido ningún umbral de intensidad mínima.

- Otros fenómenos de viento de origen convectivo: Tolvaneras, Vórtices de racha, Reventones, Frentes de racha, Reventones cálidos. Para estos fenómenos se ha establecido un umbral aproximado de intensidad mínima, de $80 \mathrm{~km} / \mathrm{h}$, ya sea viento medido o estimado. Para el caso de los reventones cálidos, el umbral se refiere al aumento de la temperatura, que debe ser (medido o estimado) de al menos $5{ }^{\circ} \mathrm{C}$.

- Vientos de ladera. Es el único fenómeno de viento no asociado a la convección que recoge el sistema. Se aplica el mismo umbral de intensidad medida o estimada de $80 \mathrm{~km} / \mathrm{h}$.

- Granizadas singulares. Definidas como aquellas en las que el granizo alcance un diámetro superior a $2 \mathrm{~cm}$, o aquellas granizadas con elementos de menor tamaño, pero que producen acumulaciones que alcancen los $2 \mathrm{~cm}$ de espesor en sitios planos y sin obstáculos cercanos.

- Precipitación súbita torrencial. Caracterizada por una duración de entre 30 minutos y tres horas, una extensión espacial inferior a $50 \mathrm{~km}^{2}$ y una cantidad de precipitación (estimada o medida) que debe ser superior a $50 \mathrm{~mm}$ en una hora en algún punto del área afectada. Este tipo de lluvias suele tener un fuerte impacto social por las inundaciones súbitas (flash flood) a las que suelen dar lugar.

- Lluvia/llovizna engelante. La precipitación engelante se considera singular en todos los casos. Es necesario aclarar que la cencellada blanca, producto de la condensación de una niebla, no es lo mismo que la cencellada transparente fruto de este tipo de precipitación, y solo este último fenómeno es el considerado en nuestro Sistema.

- Nevadas singulares. Se han establecido umbrales que caracterizan lo excepcional de la nevada para su inclusión en el Sistema.

- Aludes (Avalanchas). Solo se consideran los aludes de más de 100 m de extensión, o menores si causan daños en personas o bienes materiales.

- Fenómenos marítimos raros. Solo se incluyen aquellos de influencia meteorológica, especialmente el oleaje de rompiente y las elevaciones locales del nivel del mar.

\section{PÁGINA WEB, CARACTERÍSTICAS DE LA APLICACIÓN Y ZONAS GEOGRÁFICAS}

SINOBAS es un sistema de información geográfica colaborativo VGI (Volunteered Geographic Information), basado en Google Maps y software libre, que permite visualizar la localización geográfica de los reportes (figura 1). También queda enmarcado en el ámbito del crowdsourcing y del collaborative mapping. La dirección web de la aplicación SINOBAS es https://sinobas.aemet.es. El sistema ha sido desarrollado en AEMET y se gestiona y mantiene por personal de la propia Agencia. La aplicación ha experimentado algunas mejoras a lo largo de 2018:

- Diseño que se adapta a cualquier tamaño de pantalla y dispositivo.

- Permite al usuario subir no solo fotos y pdf, sino también videos.

- Mejora de formularios para recogida de datos y visor de la información.

- Nuevas posibilidades de interacción de los usuarios con el sistema a través de «compartir», «me gusta»y «favoritos».

- Código fuente optimizado y actualizado a nuevas versiones de PHP, MySQL, JQuery and API's.

También a destacar el hecho de que colaboran actualmente en SINOBAS, 12 asociaciones de aficionados a la meteorología, y que la divulgación de la aplicación es fundamental, algo que trata de llevarse a cabo mediante notas de prensa, cursos, conferencias, artículos en revistas y presentaciones en congresos. 
El área geográfica que se considera en SINOBAS es todo el territorio de la España peninsular y ambos archipiélagos, junto con Ceuta y Melilla. En cuanto a los fenómenos observados en el mar, el sistema admite seleccionar una ubicación hasta una distancia del orden de uno o dos kilómetro de la costa. En el caso de avistamiento de trombas marinas y tubas más lejanas, es suficiente indicar el punto desde donde se ha producido el avistamiento, junto con la dirección hacia la que se observó y la distancia aproximada.



Figura 1. Vista general de la aplicación SINOBAS en https://sinobas.aemet.es.

Para introducir información en SINOBAS, el usuario debe estar registrado. El registro se realiza, muy sencillamente, a través del enlace «Registrarse» situado en la parte superior derecha de la página principal, introduciendo unos breves datos de identificación obligatorios. Una vez cumplimentada esta información, el usuario recibe un mensaje en el correo electrónico facilitado para que confirme y finalice adecuadamente el registro. También pueden rellenarse algunos campos opcionales para completar el perfil de cada usuario.

Antes de reportar un evento, el usuario registrado debe pulsar el botón «Publicar un reporte», y posteriormente se pueden aportar dos tipos de información: información básica obligatoria e información complementaria optativa. La aplicación cuenta con un sistema guiado de introducción de datos que facilita dicha labor a los usuarios (figura 2). La información básica es la necesaria para caracterizar el evento, por ejemplo, el tipo de fenómeno y su localización temporal y geográfica, que son datos de introducción obligatoria. La información complementaria, de carácter voluntario, enriquece el reporte con datos relativos a la intensidad del evento, referencias a daños y costes económicos, información gráfica, informes de otros organismos, recortes de prensa, enlaces, u otro tipo de información relevante.

Para una mayor información acerca de algunas particularidades de la aplicación, y de los tipos de fenómenos considerados en SINOBAS, disponemos en la propia aplicación, de dos enlaces fundamentales y de recomendada consulta:

- FAQ (consultas frecuentes sobre el funcionamiento de la aplicación), https://sinobas.aemet.es/ index.php?pag=faqs.

- Guía de fenómenos, https://sinobas.aemet.es/objetos/Guia_fenomenos_sinobas.pdf, descriptiva de los fenómenos recogidos en el Sistema. 


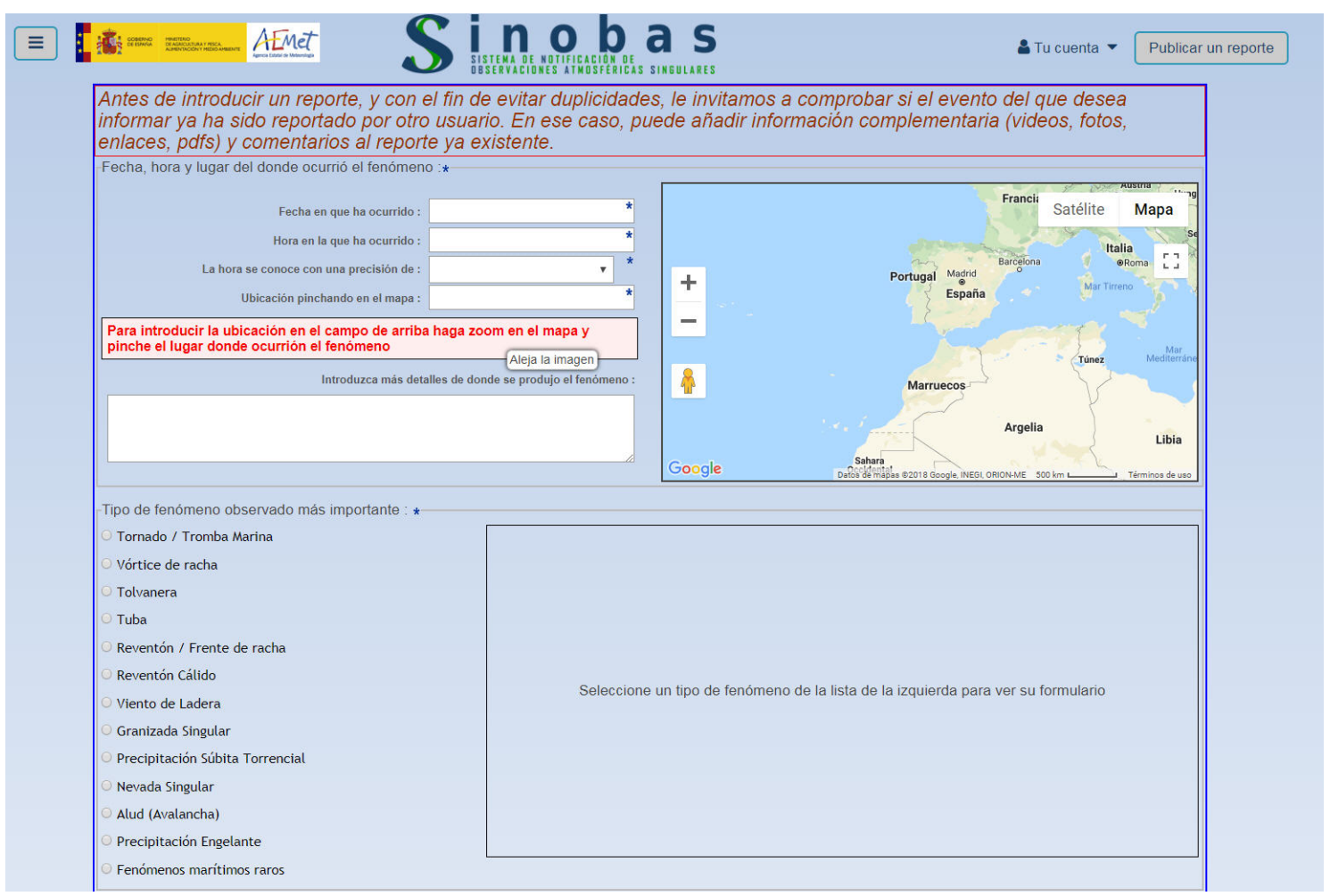

Figura 2. Introducción guiada de un reporte de tornado en SINOBAS.

En la figura 3 se muestra un ejemplo de visualización de un reporte introducido por un usuario.

En SINOBAS tenemos distintos tipos de usuarios: básicos, registrados, colaboradores, privilegiados y superusuarios.

- Los usuarios básicos son todos aquellos que usan SINOBAS para realizar consultas o visualizar fenómenos singulares, sin registrarse. La consulta a SINOBAS es libre y gratuita.

- Los usuarios registrados son aquellos que se registran e identifican, pueden subir reportes y dejar comentarios pero no pueden modificar ni borrar datos ya existentes.

- Los usuarios colaboradores son miembros de organizaciones externas a AEMET (asociaciones de aficionados, organismos oficiales, etc.) con las que se ha establecido una colaboración. Existe un usuario colaborador por cada organización colaboradora. Este usuario puede invitar a otros miembros de su organismo a formar parte de su grupo, y es responsable de las validaciones de los reportes introducidos por dicho grupo.

- Los usuarios privilegiados son personal de AEMET que dispone de acceso a cualquier tipo de reporte, y pueden modificar la información y validar los reportes, adjudicándoles una fiabilidad determinada.

- Los superusuarios constituyen un reducido grupo de personas de AEMET, encargadas de gestionar, administrar y coordinar la operatividad del Sistema.

Todo reporte que se registra en SINOBAS, aparece inicialmente como no validado. Una vez que ha sido analizado y validado, se le adjudica una fiabilidad (baja, media o alta —en el caso, muy poco frecuente, de que el validador considere imposible verificar la información se clasifica como imposible de validar-), que será asignada por los usuarios privilegiados o, en su caso, por los representantes de las asociaciones colaboradoras. Los reportes muy poco verosímiles, considerados de fiabilidad nula, son eliminados del sistema abierto al público, aunque quedan archivados en la base de datos de AEMET. 


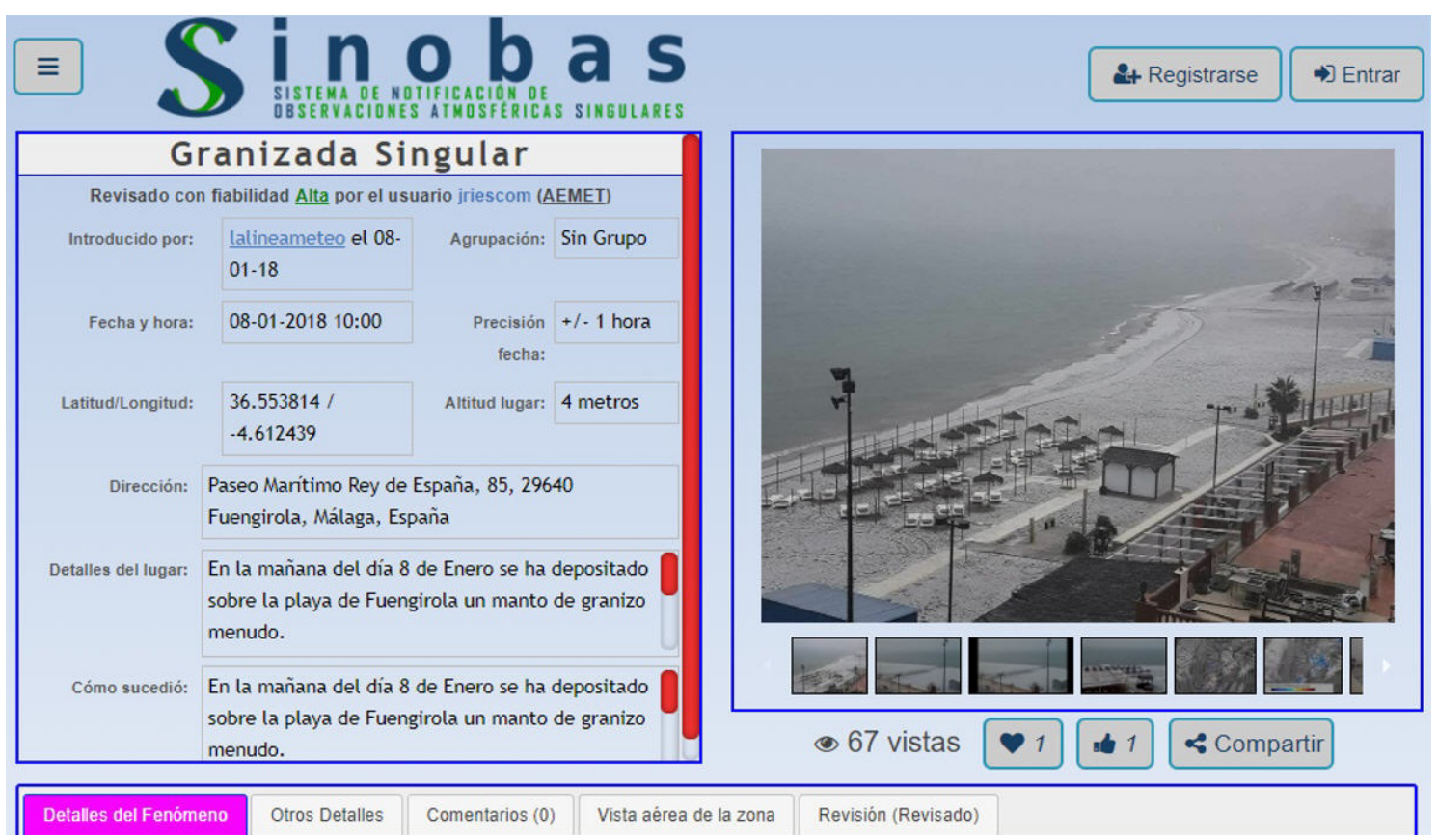

Figura 3. Ejemplo de vista resumida de un reporte archivado y validado en SINOBAS.

\section{SINOBAS COMO PRIMER ESCALÓN DE VIGILANCIA}

Además la herramienta SINOBAS cuenta con dos herramientas internas (solo para personal registrado con el correo electrónico de AEMET), que pueden servir como primer escalón de vigilancia meteorológica, pues permiten captar información de redes sociales de contenido propiamente meteorológico.

\subsection{Rastreo de cuentas Twitter}

La primera opción, configurada desde el panel de administración de la aplicación SINOBAS, es la del rastreo de ciertas cuentas de Twitter (seleccionadas de entre el «mundillo meteorológico»). Lo que se busca en estas cuentas es la aparición de ciertas palabras de interés, como por ejemplo tormenta, niebla, precipitación, etc.

Existen dos filtros de búsqueda configurables: cuentas de Twitter y palabras a buscar. Tras la búsqueda aparecerán los tuits en una zona de la pantalla y los filtros por ambas categorías en un listado accesible (figura 4). Así se puede disponer internamente de información valiosa de lo que está pasando en tiempo real, especialmente en el aspecto de la fenomenología observada en superficie (tormenta, niebla, precipitación intensa, etc.). También pueden aparecer tuits que nombran o contienen referenciadas las cuentas de Twitter que tenemos por defecto seleccionadas.

\subsection{Rastreo de foros meteorológicos}

La segunda opción, también configurable desde el panel de administración de SINOBAS, se refiere al rastreo de foros. Concretamente se puede acceder a información publicada en los foros http://foro.tiempo.com/y http://www.cazatormentas.net/foro.

En ambos aparece información que puede resultar muy valiosa, si se filtra adecuadamente la búsqueda mediante un grupo de palabras de tipo meteorológico bien seleccionadas. Los resultados de la búsqueda aparecen ordenados cronológicamente en la parte central de la página, mientras que a la derecha aparecerán las entradas de los foros, accesibles por categorías, según la palabra, tema, foro/subforo e información complementaria. Un ejemplo que muestra la salida gráfica de esta utilidad se presenta en la figura 5. 


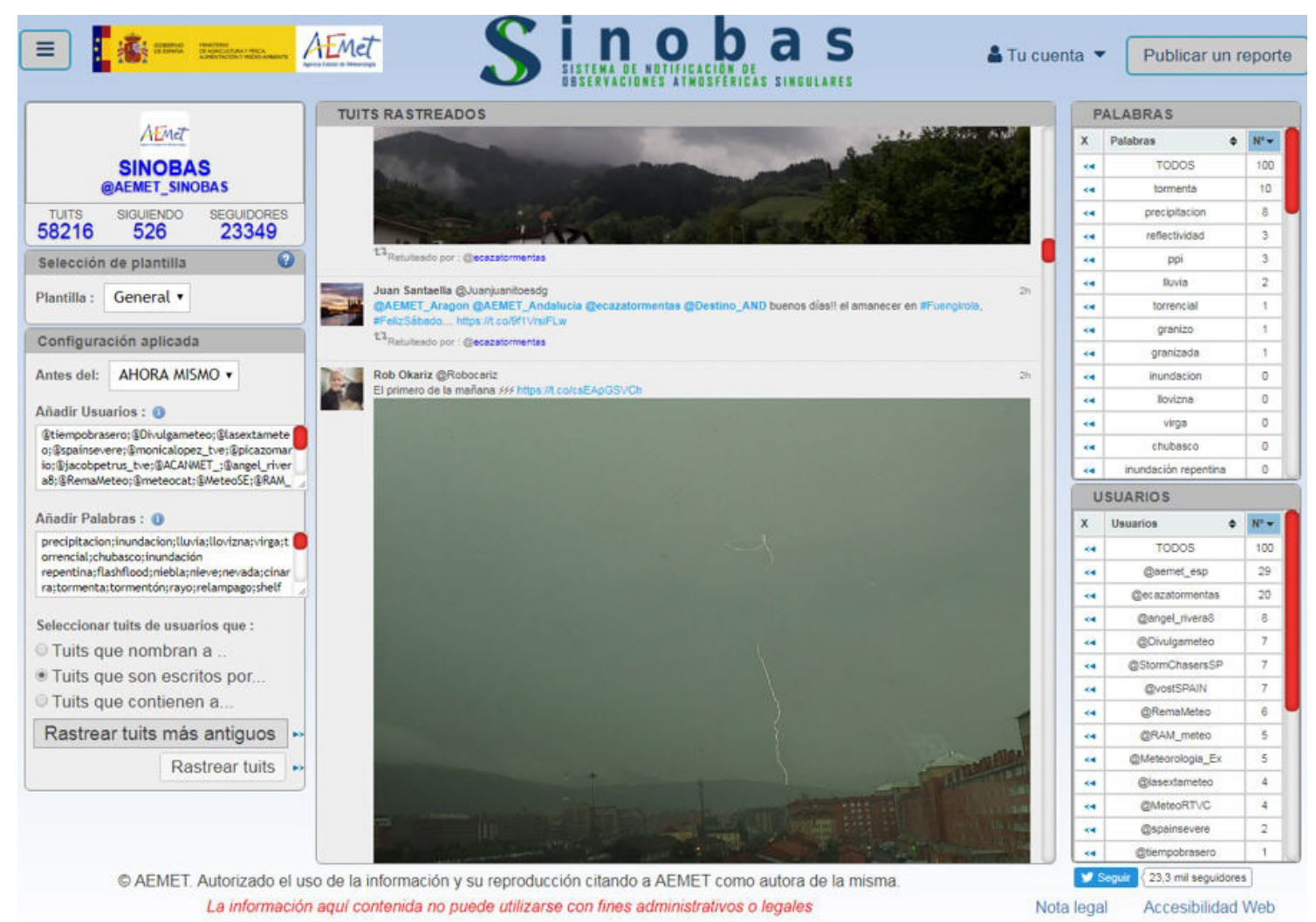

Figura 4. Ejemplo de rastreo de cuentas de Twitter en SINOBAS.

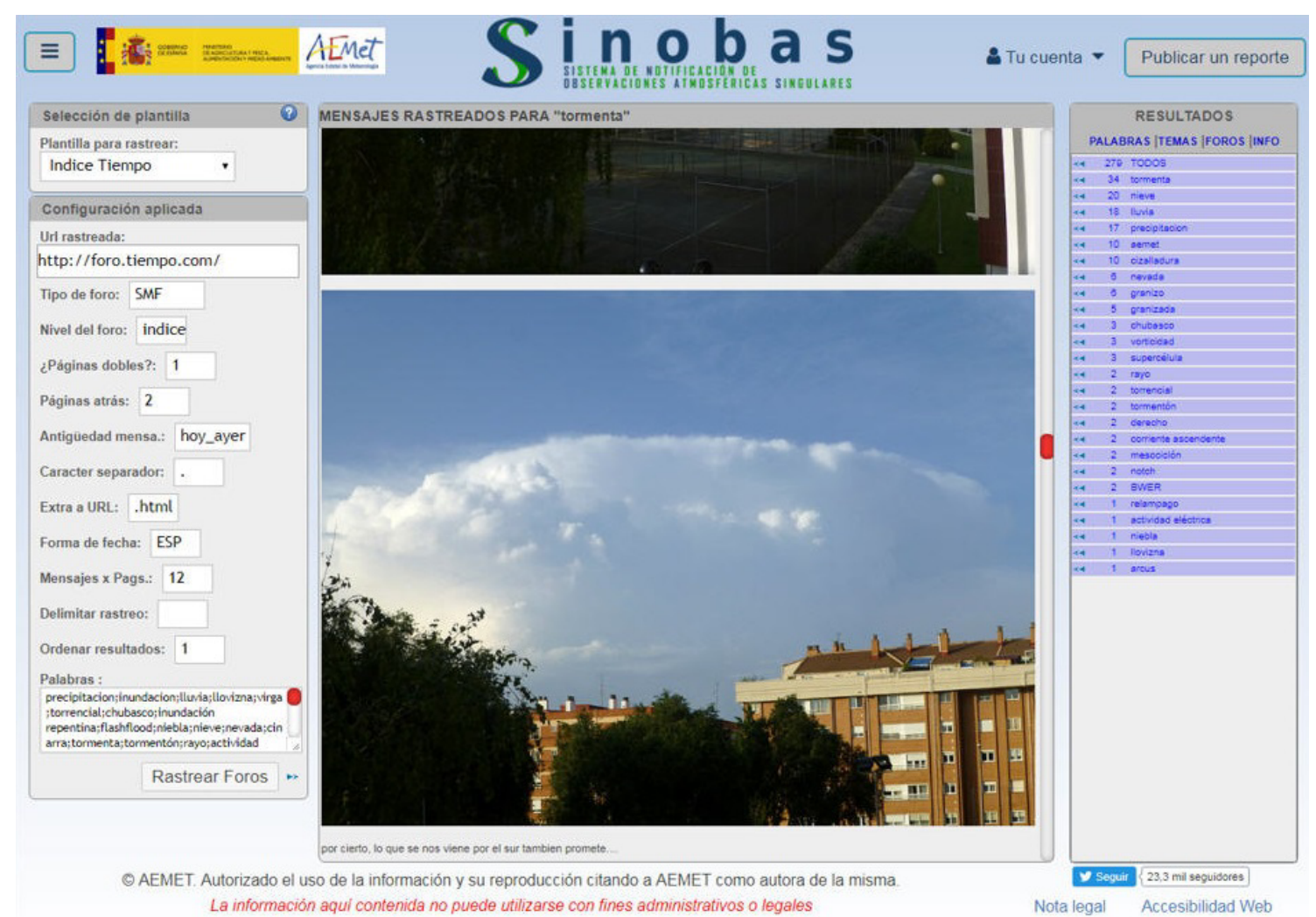

Figura 5. Ejemplo de rastreo de foros en SINOBAS. 


\section{ESTADÍSTICAS}

SINOBAS se abrió al público en abril de 2013, y en estos ya más de 5 años se ha ido consolidando como una referencia entre los aficionados e interesados en la meteorología, lo que redunda en el crecimiento continuo de nuestra base de datos de eventos, tanto históricos como actuales.

A fecha de 31 de agosto de 2018, contamos con más de 1400 usuarios registrados en la aplicación SINOBAS, en torno a 1100 reportes de tiempo singular y con más de 350000 visitas al sitio web.

La mayoría de los reportes corresponden a eventos ocurridos en fechas recientes, siendo nuestro objetivo que cualquier fenómeno singular del que se tenga constancia sea registrado en SINOBAS a la mayor brevedad, bien por los testigos directos, o por cualquier ciudadano que tenga conocimiento del evento. No obstante, también es muy valioso el enriquecimiento del sistema con la introducción de reportes de eventos históricos, y en este sentido, tenemos que agradecer la inestimable colaboración de algunos usuarios que han volcado sus datos en nuestro sistema.

En cuanto a estadísticas básicas (figura 6), cabe destacar que los tipos de fenómenos más reportados son los tornados/trombas marinas, seguidos del granizo singular y las precipitaciones súbitas torrenciales. La inmensa mayoría de reportes son validados con fiabilidad alta por los profesionales de AEMET. Los usuarios que más reportes incluyen son aficionados particulares, responsables de AEMET y también destacan en este apartado las asociaciones de AMETSE y ACAM.

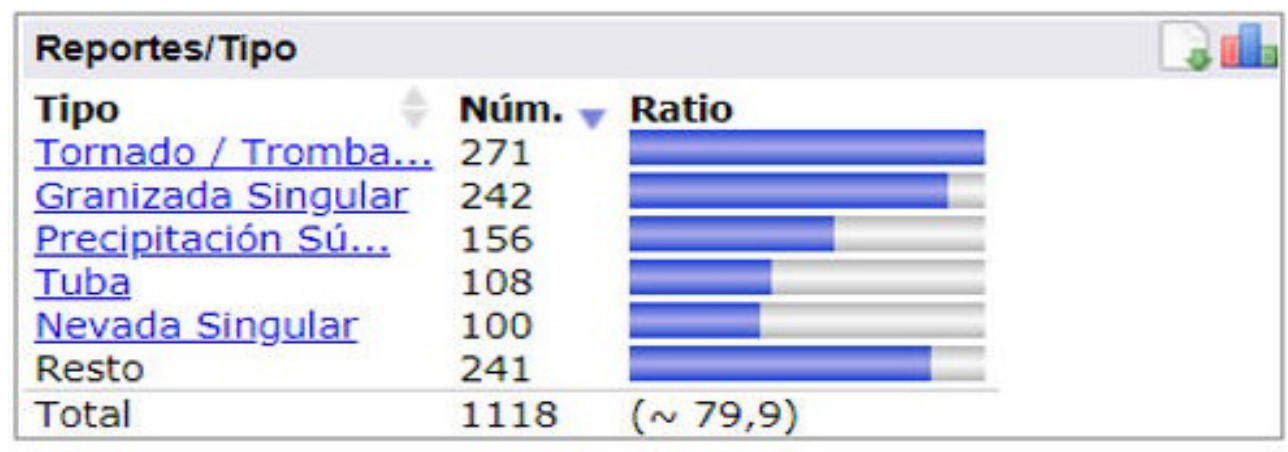

\begin{tabular}{|lll|l|}
\hline \multicolumn{2}{l}{ Reportes/Grupo } \\
Grupo & Núm. $\mathbf{v}$ Ratio \\
\hline NINGUNO & 619 & \\
\hline AEMET & 396 \\
\hline AMETSE & 47 \\
\hline ACAM & 33 & \\
\hline AVAMET & 7 & \\
\hline Resto & 16 & \\
\hline Total & 1118 & $(\sim 101,6)$ \\
\hline
\end{tabular}

\begin{tabular}{|lll|}
\hline Reportes/Fiabilidad & & \\
Fiabilidad & Núm. $\mathbf{~ R a t i o ~}$ \\
\hline Alta & 899 & \\
\hline Media & 77 & \\
\hline Eliminado & 51 & \\
\hline No Validado & 47 & \\
\hline Baja & 28 & \\
\hline Imposible de val... & 16 & \\
\hline Total & 1118 & $(\sim 186,3)$ \\
\hline
\end{tabular}

Figura 6. Algunas estadísticas complementarias de SINOBAS. 
Adicionalmente, es importante resaltar que, desde marzo de 2014, existe una cuenta oficial de Twitter asociada a SINOBAS (@AEMET_SINOBAS), que cuenta a fecha de 31 de agosto de 2018 con más de 25000 seguidores. Esta cuenta, que difunde información relativa a los reportes y las validaciones, así como información meteorológica básica, y especialmente la relacionada con los fenómenos meteorológicos recogidos por el sistema, se ha demostrado como una valiosa herramienta de popularización del sistema y de comunicación con nuestros usuarios.

\section{CONCLUSIONES}

Mediante la aplicación web SINOBAS (https://sinobas.aemet.es), AEMET recoge información de cualquier ciudadano sobre la ocurrencia de fenómenos meteorológicos que, por su escala, pueden pasar desapercibidos para las redes de observación convencional y para los sistemas de teledetección, pero que tienen relevancia y pueden causar un impacto significativo en la población, como por ejemplo tornados, granizadas singulares, precipitaciones súbitas torrenciales, nevadas singulares, etc.

Actualmente la aplicación web, desarrollada en AEMET, ha renovado su estética y algunas funcionalidades. Está basada en software libre y referenciada geográficamente sobre Google Maps. Cuenta con cerca de millar y medio de usuarios registrados y más de mil fenómenos meteorológicos «singulares» reportados desde su puesta en operación. Todos los reportes introducidos en el sistema son después validados por técnicos de AEMET y se les asigna una fiabilidad.SINOBAS también gestiona una cuenta de Twitter, @AEMET_SINOBAS, que es un canal ágil de comunicación con el público y una vía de popularización del sistema y seguimiento de la actualidad meteorológica.

Por último es destacable la capacidad de vigilancia en un primer escalón, de las aplicaciones internas que ofrece SINOBAS de rastreo meteorológico en Twitter y en foros de aficionados. Ambas sirven para recibir información sobre fenómenos meteorológicos relevantes en tiempo real.

\section{AGRADECIMIENTOS}

Es justo dar las gracias a todos los ciudadanos que con su colaboración dan sentido a nuestro Sistema, y comparten con nosotros valiosa información, enriquecida en ocasiones con hermosas fotografías y, casi siempre, con buenas dosis de emoción y entusiasmo. Animáis cada día nuestro trabajo con vuestros reportes y vuestros tuits. ¡Un millón de gracias!

\section{REFERENCIAS}

Gutiérrez, D., Riesco, J., Díez, E., Martín, F., NúÑez, J. A., Sánchez-Laulhé, J. M. y Ferri, M., 2013. Breve guía descriptiva de los fenómenos meteorológicos recogidos en el Sistema de Notificación de OBservaciones Atmosféricas Singulares. AEMET. https://www.aemet.es/documentos/es/eltiempo/observacion/ sinobas/breve_guia_descriptiva_SINOBAS.pdf.

European Severe Weather Database. https://www.eswd.eu/. 
